\title{
Patient with osteoarthritis, aggravated by focal infection from dental origin-a case report
}

\author{
Assya Krasteva ${ }^{1}$, Yanitsa Istatkova ${ }^{1}$, \\ Mirela Marinova-Takorova ${ }^{2}$
}

1. Department of Oral Imaging and Oral Diagnostics, Faculty of Dental Medicine, Medical University Sofia, Bulgaria

2. Department of Conservative Dentistry, Faculty of Dental Medicine, Medical University - Sofia, Bulgaria;

\begin{abstract}
Osteoarthritis is a degenerative joint disease, affecting approximately 27 million Americans. It can affect any joint, but it occurs most often in knees, hips, lower back and neck, small joints of the fingers and the bases of the thumb and big toe.

We present a 53 years-old woman with chronic OA of the small joints of the fingers with complaints of pain, swollen and warmed joints of the left and right hands. The patient was referred to the Department of Oral Imaging and Oral Diagnostics to perform a consultation due to elevated levels of CRP and ESR. The patient underwent focal dental diagnostic protocol. We remarked an active dental focustooth 46. It was retreated endodontically under antibiotic protection and the patient was followed-up after one month with decreased levels of CRP and ESR.
\end{abstract}

Keywords: osteoarthritis, dental focal infection, CRP, ESR. 


\section{Background:}

Osteoarthritis (OA) is the most common joint disorder in the United States, affecting 10\% of men and $13 \%$ of women aged 60 years or older. The number of people with symptomatic osteoarthritis is likely to increase due to the aging of the population and the obesity (1). Epidemiological data relieves information that in the United Kingdom nearly $10 \%$ of the population has osteoarthritis. The most common symptoms are pain, stiffness and severe disability (2).

Pain from OA is a key symptom in the decision to seek medical care and is an important antecedent to disability. Because of its high prevalence and the frequent disability that accompanies disease in major joints such as the knee and hip, OA accounts for more difficulty with climbing stairs and walking than any other disease (1).

Usually, the clinical symptoms of osteoarthritis are overviewed by different laboratory tests but most authors prefer to investigate erythrocyte sedimentation level and C- reactive protein.

C-reactive protein (CRP) is an acute-phase protein produced by the liver. Its levels rise dramatically during inflammation that occurs in the body. This increment of CRP is due to a rise in the plasma concentration of interleukin-6, produced predominantly by macrophages and adipocytes. During the acute phase response, CRP levels increase rapidly within 2 hours, CRP declines with a half-life of 18 hours. CRP can rise up to 50000 fold in acute inflammation, such as during infection (3).

Different studies have demonstrated an association between osteoarthritis progression and inflammation as measured by systemic C-reactive protein levels (4).

Xingzhong and co-workers have found that levels of CRP were significantly associated with pain and decreased physical function (5).

The erythrocyte sedimentation rate is a surrogate marker of the acute phase reaction. During an inflammatory reaction, the sedimentation rate is affected by increasing concentrations of fibrinogen, the main clotting protein, and alpha globulins. The test mainly measures the plasma viscosity by assessing the tendency for red blood cells to aggregate and 'fall' through the variably viscous plasma (6).

Normalisation of an elevated erythrocyte sedimentation rate, once an immunoglobulin response has occurred, may take weeks to months.

Raised erythrocyte sedimentation rates are observed in patients without an acute phase reaction, for example when haematological disorders including anaemia are present. Renal failure, obesity, ageing and female sex are associated with higher erythrocyte sedimentation rates. C-reactive protein results are also higher with obesity but are not affected by renal failure (6).

F. Wolfe has investigated association between CRP and ESR in their ability to associate with osteoarthritic symptoms and features. The author has found that the median value of CRP was 5.9 micrograms $/ \mathrm{ml}$ and CRP was significantly associated with functional disability, joint tenderness, pain, fatigue, global severity, and depression. ESR, by contrast, was unassociated with clinical signs or symptoms except for a weak association with functional disability (7). 


\section{Case description:}

We present a 53 years old woman with chronic OA of the small joints of the fingers with complaints of pain, swollen and warmed joints of the left and right hands. The patient was referred to the Department of Oral Imaging and oral diagnostics to perform a consultation due to elevated levels of CRP and ESR. The patient complained of painful, swollen and warmed joints - of the middle finger of the right hand and the joint of the thumb of the left hand (Fig.1).

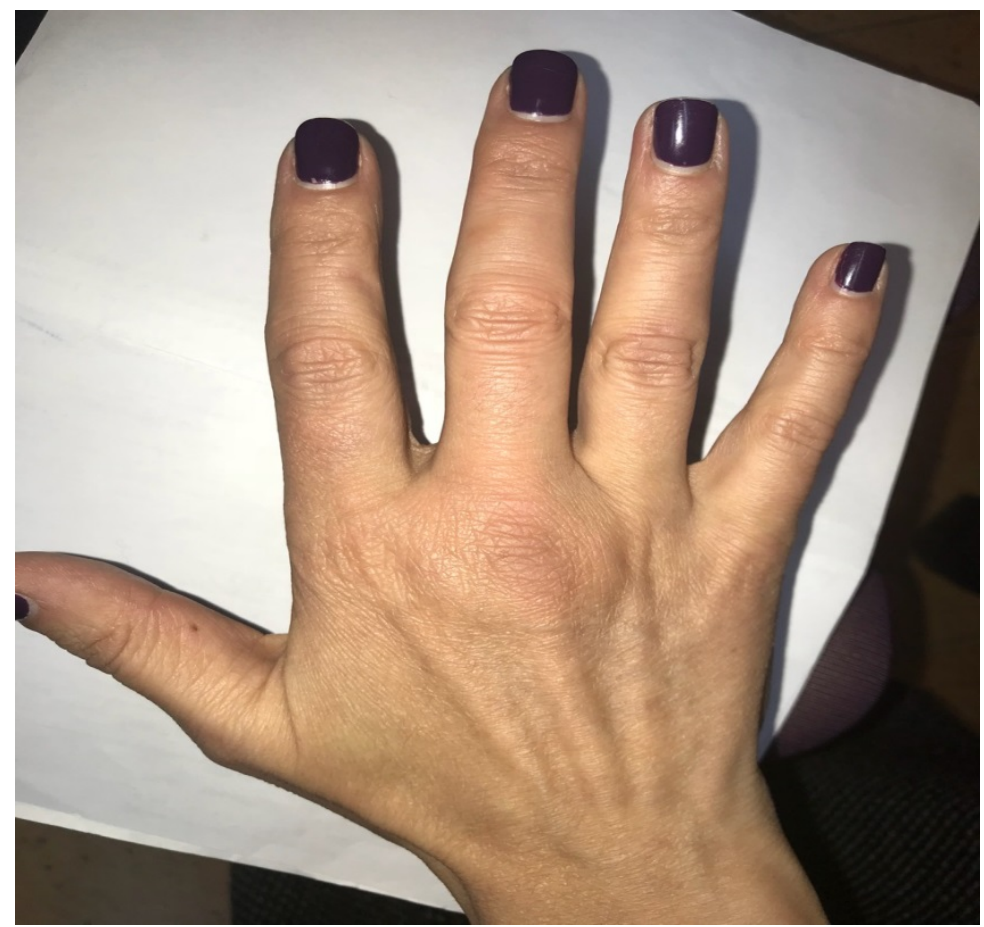

Fig.1

The patient didn't have any complaints from the oral cavity and teeth. She underwent focal diagnostic protocol which included:

1. Clinical examination - dental status, palpation of lymph nodes, percussion;

2. X-ray diagnostics - ortopantomography;

3. Gelen test (electro skin test) - for detection active or potential dental foci;

4. Electroodontodiagnostics - test for the vitality of the teeth;

5. Thermovision research during Flir A360 in 6 aspects.

6. Complete blood count with differential (CBC), including additionally levels of blood glucose, creatinine, uric acid, CRP and ESR.

Thermography is not invasive to the human body and is not-contact technique through which the temperature distribution of the human body surface could be monitored and recorded- physiological asymmetry to- 0.4; 


\section{Results}

1. We remarked active dental focus - tooth 46; based on the results from test of Gelen, and Thermography

2. EOD reveal: tooth $16=11 \mu \mathrm{A}$; tooth $26=14 \mu \mathrm{A}$; tooth $37=10 \mu \mathrm{A}$; tooth $36=10 \mu \mathrm{A}$;

3. X-ray: data for canal fillings with no good quality of tooth 46 .

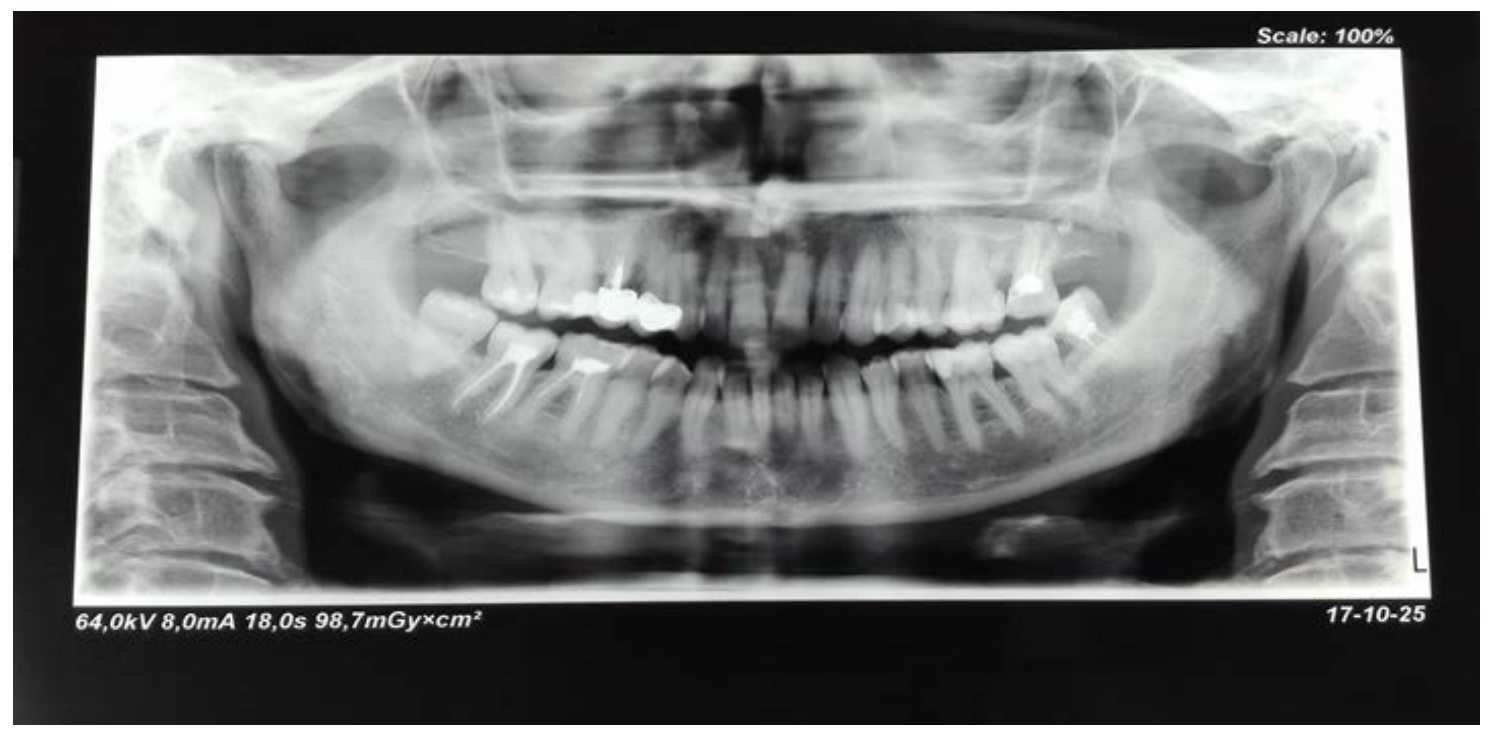

Fig. 2 Diagnostic ortopantomography

4. As potential dental foci we remarked - teeth $15,27,38,47$; based on the result from test of Gelen

5. The levels of $E S R=30 \mathrm{~mm} / \mathrm{h}$ (normal value $=<25 \mathrm{~mm} / \mathrm{h}$ ); CRP $=4.86 \mathrm{mg} / \mathrm{l}$ (normal value $=<5 \mathrm{mg} / \mathrm{l}$ ).

\section{DENTAL RECOMMENDATIONS:}

Retreatment of the active dental focus under antibiotic protection with Azatryl $500 \mathrm{mg}$ (1 day before; at the day of conservative treatment -1 hour before that; 1 day after the endodontic treatment).

The clinical diagnosis of the tooth 46 was Periodontitis chronica granulomatosa diffusa. Working length was determined using C-pilot files (WDW Germany). The canals were enlarged using ProTaper universal files (Meileffer) till size F3. The irrigation protocol included $5.25 \% \mathrm{NaOCl}, 3 \% \mathrm{H} 2 \mathrm{O} 2$ and EDTA. Root canals were filled with Seal Apex using calibrated gutta-percha points till size F3 (Fig. 3). 


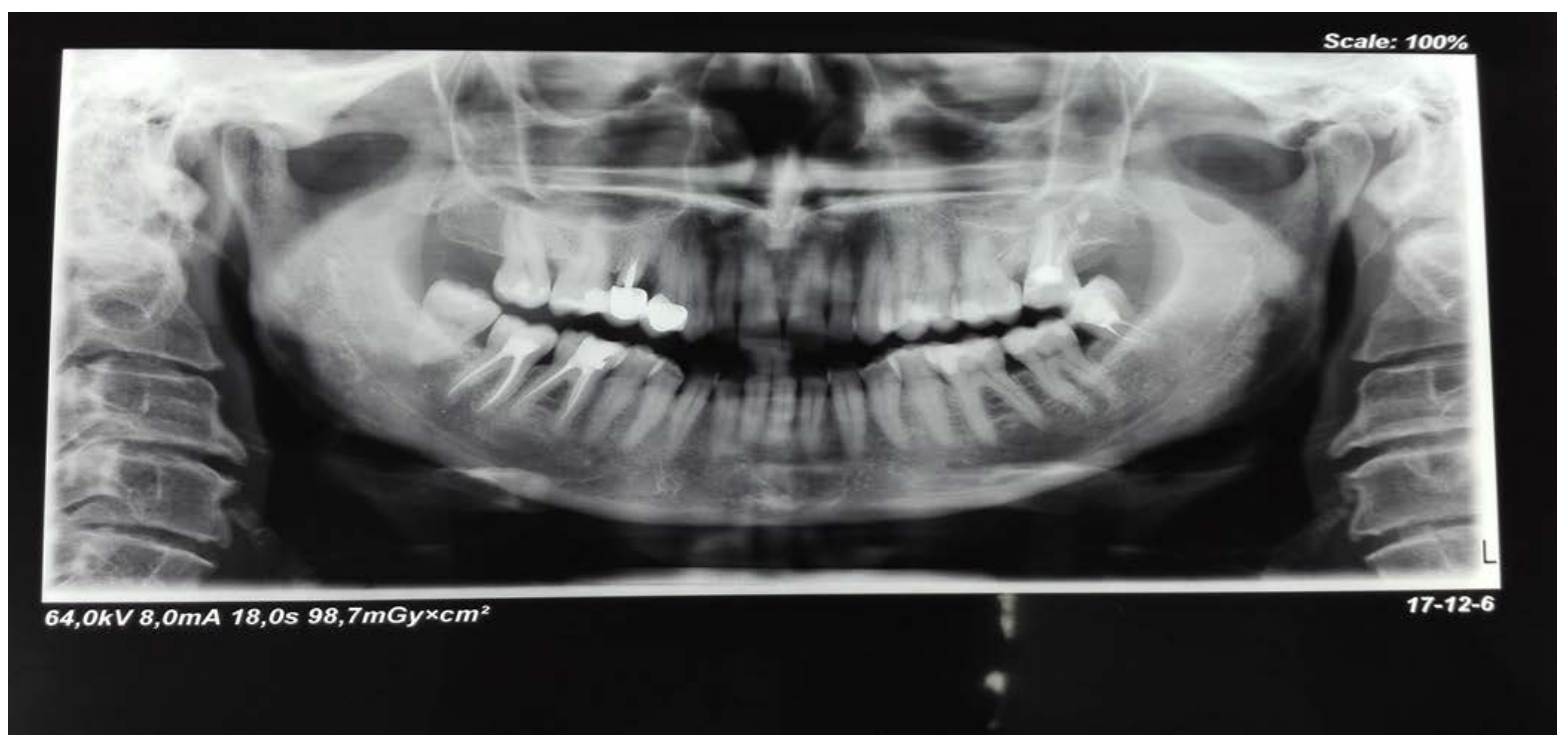

Fig. 3 Ortopantomography after endodontic treatment of tooth 46

\section{Discussion}

In recent years there has been a reawakening of the dangers of oral infections and their potential disastrous effects on systemic health.

"The detrimental effect of local infection on general health has been known for decades. Chronic dental infections may worsen the condition of medically compromised patients" (8).

The theory of focal infection, which was promulgated during the 19th and early 20th centuries, stated that "foci" of sepsis were responsible for the initiation and progression of a variety of inflammatory diseases such as arthritis, peptic ulcers and appendicitis. In the oral cavity, therapeutic edentulation was common as a result of the popularity of the focal infection theory. A number of epidemiological studies have suggested that oral infection, especially marginal and apical periodontitis, may be a risk factor for systemic diseases.

The teeth are the only no shedding surfaces in the body, and bacterial levels can reach more than $10^{11}$ microorganisms per mg of dental plaque. Human endodontic and periodontal infections are associated with complex microflora in which approximately 200 species (in apical periodontitis) and more than 500 species (in marginal periodontitis) have been encountered (9).

The incidence of bacteriemia following dental procedures such as tooth extraction, endodontic treatment, periodontal surgery, and root canal scaling has been well documented. Bacteriemia was observed in $100 \%$ of the patients after dental extraction, in $70 \%$ after dental scaling, in $55 \%$ after third- molar surgery, in $20 \%$ after endodontic treatment, and in $55 \%$ after bilateral tonsillectomy. 
Three mechanisms or pathways linking oral infections to secondary systemic effects have been proposed. These are metastatic spread of infection from the oral cavity as a result of transient bacteremia, metastatic injury from the effects of circulating oral microbial toxins, and metastatic inflammation caused by immunological injury induced by oral microorganisms (9).

There are different articles in general medicine describing the relation between focal infections and the general health of the patients. Different authors have presented several clinical cases with systemic diseases and improved general symptoms after treatment of the oral and focal infections.

A Krasteva and co-workers have described a clinical case of a hepatitis B patient with fever due to a focal infection. The patient had progressive hypoalbuminemia and elevated CRP. The patient underwent consultation with endocrinologist, rheumatologist, neurologist, gynecologist, pulmonologist and infectionist. All the results were negative. Only oral examination revealed active dental foci and dental recommendation was extraction of the proven teeth. The teeth were extracted under antibiotic treatment with Clindamycin and the patient became afebrile 5 days after the last extraction. Her liver enzymes, CRP and albumin returned to normal (10).

AC Breebaart and co-workers have reported a clinical case of a patient with remission of rheumatoid arthritis of 16 years duration caused by the extraction of endodontically well-treated, healthy looking teeth. After extraction the rheumatoid factor became negative and the patient remained symptom - free for the next 16 years. The possible connections between microorganisms in closed dental foci under constant pressure and the chronicity and exacerbations of rheumatoid arthritis are proven in this clinical case (11).

An author, Gerald Smith, has presented a clinical case of a 64 years-old female patient with Rheumatoid arthritis who was on severe immunosuppressive therapy (corticosteroids, Prednisone, chemotherapy drugs, Methotrexate and a deactivating drug- Enbrel). Following two treatment sessions, MT's rheumatoid arthritis resolved by $90 \%$ and she stopped taking the Enbrel. No additional therapy was instituted at the time of treatment. This patient has regained a pain- free quality of life. The key factor was the removal of the underlying cause- infected root canal tooth- which started the release of the tumor necrosis factor (12).

The presented clinical case also confirms the connection between dental foci and systemic diseases, particularly osteoarthritis. After dental treatment of the active dental focus, the patient's complaints are resolved and the abnormal laboratory tests became normal (levels of ESR $=18 \mathrm{~mm} / \mathrm{h} ; \mathrm{CRP}=0.52 \mathrm{mg} / \mathrm{l}$ ). After the dental treatment the patient doesn't take any systemic medications for the general disease.

\section{Conclusion}

It has always been important for patients with systemic and general diseases to perform regular focal diagnostics according to the generally accepted protocol because the presence of foci of oral and dental origin may influence the clinical manifestation of the main disease, additionally it could interfere with some blood parameters, which are characteristic for inflammatory diseases.

The presented clinical case is an example of a favorable response of the clinical manifestation and normalization of the levels of CRP and ESR after the treatment of dental foci. 


\section{References}

1. Zhang Y, Jordan JM. Epidemiology of osteoarthritis. Clin Geriatr Med 2011; 26:3:355-369.

2. Hiligsmann M, Reginster JY. The economic weight of osteoarthritis in Europe Medicographia 2013;35:1

3. Seo HS. The role and clinical significance of high-sensitivity C-reactive protein in cardiovascular disease. Korean Circ J 2012;42:3: 151-153

4. Pearle AD, Scanzello CR, George SS, et al. Elevated C-reactive protein levels in osteoarthritis are associated with local joint inflammation. Arthritis Res Ther 2004; 6:3:56

5. Jin X, Beguerie JR, Zhang W, et al. Circulating C-reactive protein in osteoarthritis: a systematic review and meta-analysis. Annals of the Rheumatic Diseases 2015;74:703-710

6. Harrison M .Erythrocyte sedimentation rate and C-reactive protein. Aust Prescr. 2015; 38:3: 9394

7. Wolfe $\mathrm{F}$. The C-reactive protein but not erythrocyte sedimentation rate is associated with clinical severity in patients with osteoarthritis of the knee or hip. The Journal of Rheumatology 1997;24:8:1477-1485

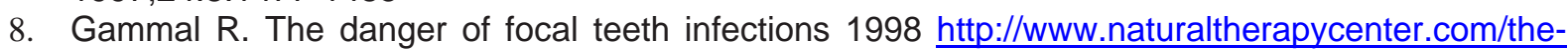
dangers-of-focal-teeth-infections/

9. Li X, Kolltveit KM, Tronstad L, et al. Systemic diseases caused by oral infection. Clin. Microbiol. Rev. 2000 ;13:4 547-558 1

10. Krasteva A, Panova D, Antonov K, et al. Hepatitis B patient with fever due to focal infection. J of IMAB 2012; 18:4:251-254.

11. Breebaart AC, Bijlsma JW, van Eden W. 16-year remission of rheumatoid arthritis after unusually vigorous treatment of closed dental foci. Clin Exp Rheumatol 2000;20:4:555-557.

12. Smith GH. Dental/Rheumatoid arthritis Connection http://www.icnr.com/cs/cs 23.html

\section{Corresponding author:}

Dr Yanitsa Istatkova,

Department of Oral Imaging and oral diagnostics,

Faculty of Dental medicine,

Medical University - Sofia, Bulgaria,

University Hospital "Dr G.Stranski",

1, bul. Sv. Georgi Sofiiski,

email: q_a_istatkova@abv.bg 